\title{
On The Stratified Structure Of Solar Active Regions
}

\author{
A.A. Galal \\ National Research Institute of Astronomy and Geophysics (NRIAG) \\ Dr- FATTAH @ Yahoo. Com
}

\begin{abstract}
The manuscript presents a review of the results obtained by the author from fast sequential H-alpha filtergrams taken for a quiescent filament together with similar results of other authors. The revealed structure clearly shows that the foot elements of the filament are surrounded by a fishbone-like plage area (magnetic region). Some parts of the filament exist at the boundary of large supergranular cells of about 40" in diameter. It seems that the filament is formed due to mixing of magnetic elements of different polarities.
\end{abstract}

Keywords. H-alpha, filament, magnetic region

\section{Introduction}

Investigations of quiet and active regions of the solar atmosphere date back to the time of the invention of the spectroheliograph by G. Hale in 1891. Further observational progress came after the invention of birefringet filter by Lyot in 1933 and in 1937 by Ohmann. Due to limited space the reader is referred to monograph given by Loughead and Bray (1974), Athay (1976) and the proceedings edited by Bonnet and Delache (1976) and by Ulmschneider et al. (1991). During the last decade, rich harvest came from the SOHO Spacecraft (Harison et al. 1995; Domingo et al., 1995; VIRGO group, 1995; Fleck et al. 1995; Kenneth R. Lang, 2001). Most of the studies of the formation and development of dark solar filaments have been devoted to the development of filaments with time as shown in the H-alpha line center. (Smith, 1968, Plocieniak and Rompolt; 1972, Martin, 1973; 1986; Herman and Martin, 1986) A rapid sequence of H-alpha filtergrams for the solar disk have been secured on $23^{\text {rd }}$ October, 1981, using the automatic H-alpha monochromator adapted to the 150/225 (cm) Coude refractor of King Abdul Aziz University, Saudi Arabia. The system enables to take fast series of spectral solar photographs up to 400 exposures without any manual intervention. Preliminary investigations of the data indicate obvious variability of the structure of the examined filament in transition from the photosphere to the chromospheric (Galal, 1983; 1989). In what follows we shed the light on the magnetic structures and polarities located at the levels of the solar atmosphere where dark filaments are originated.

\section{Proto-Elements of the filament}

Plate I exhibits the recorded changes in the structure of the observed filament in transition from the center to the blue wing of $\mathrm{H}$-alpha. In other words these records illustrate the stratified structure of the ascending parcels of the solar plasma in the filament. It is easy to notice in the filtergrams taken at displacements $\Delta \lambda=0.45 \mathrm{~A}^{\circ}$ and $0.5 \mathrm{~A}^{\circ}$ that the proto-elements of the filament are isolated from the adjacent chromospheric net work by elongated plage area ( enhanced region of magnetic field ) having exactly the same shape of the filament. One may hardly distinguish between the just appearing filamentary 
strands and the neighboring intergranular spaces. The pattern of plage elements seen in these pictures distinctly indicates that the examined filament is built on a fishbone - like magnetic field.

\section{Inversion of Polarities}

Inspection of the Stanford magnetogram taken at $22 \mathrm{~h}: 06 \mathrm{~m}$ UT on $23^{\text {rd }}$ October, 1981 (Solar - Geophysical Data, prompt reports, December, 1981 No 448 - Part 1) indicates inversion of the magnetic polarity on both sides of the filament (cf. Fig. 1b). It appears therefore more likely that the interaction between plage areas (magnetic regions) of opposite polarities leads to the formation of dark filaments. The magnetic field pattern above the existing bipolar magnetic region involves a sheet pinch and a neutral line (Furth et al, 1963, Sturrok, 1968). In addition, the mixing of opposite polarity line of sight magnetic elements also leads to the cancellation and disappearance of these elements (Martin, 1986). These circumstances are considered vital pre-existing causes for the formation of dark filaments.

\section{Signs for Large-scale Motions}

The distribution and orientation of the plage (magnetic region) protrusions with respect to the filament imply that the filament region is subjected to a large scale mass motion. The differential effects of active regions in the vicinity of the filament (cf. Big Bear map, Fig. 1a) may be responsible for exerting large scale motion giving rise to mixing of magnetic regions of opposite polarities. The first and second pictures in Plate I give a general impression about the existence of a large - scale horizontal shear flow with a converging velocity component towards the axis of the filament (neutral line).

\section{Link with large Supergranules}

The different filtergrams in plate I generally show that some parts of the filament are segments of the boundaries of relatively large supergranular cells $(\approx 40 ")$. The arrows point to some of these cells which boundaries are linked to the body of the filament under test . A proto-type filament may arise from the alignment of dark fibrils at the boundary of large supergranular cells. The fibrils may be sheared by mass motions to form a long H-alpha filament ( Giovanelli, 1984, Rompolt and Bodgan, 1986).

\section{Top Layers of the Filament}

As we go nearer to the center of H-alpha line (cf. Plate I, 3, 4) the whole body of the filament becomes more and more visible. The plage features become more bright and compact especially at $\Delta \lambda=0.4 \mathrm{~A}^{\circ}$, where extended bright patches are seen inside large supergranular cells with broken boundaries. The dark intergranular spaces become smoother and less dark on such filtergram. The large dark lane $(\approx 90 "$ in extension and with few faint granules) seen under the right hand curved part of the filament in filtergrams 1 and 2 is inverted into bright plage elements at $\Delta \lambda=0.4 \mathrm{~A}^{\circ}$, but the bright elements in it become a little bit less dense and bright at $\Delta \lambda=0.35 \mathrm{~A}^{\circ}$. The final form of the filament appears at $\Delta \lambda=0.35 \mathrm{~A}^{\circ}$, where the various dark segments of its body combined together and some of the gaps between them disappeared. Very close to the $\mathrm{H}$-alpha line center the recorded variations in the shape and structure of the filament are not significant. 


\section{Discussions and Conclusion}

The fast set of H-alpha filtergrams exhibited in Plate I have been taken during an interval of time about 8 seconds, i.e. the time variation of the recorded features of the filament is greatly reduced. In other words these filtergrams reflect to a great extent the stratified structure of the observed filament. The main elements configurating the filament (e.g. the plage areas and protrusions, large supergranular cells and bright bridges along the filament body) and the systematic changes in their definitions and features are obviously revealed in transitions from the foot layers of the filament to higher levels. It seems that the ejection of the solar plasma in a quiescent filament begins at the level of the chromespheric supergranules. Under the influence of the Sun's gravitational field together with hydromagnetic effects, the velocity of upward moving parcels of plasma gradually decreases to about zero- value. This simply may explain the insignificant changes in the body of the filament very close to the line center. As suggested by Forbes (1986) a great part of the material contained in a long lived filament is transported by a certain mechanism from the chromosphere. 

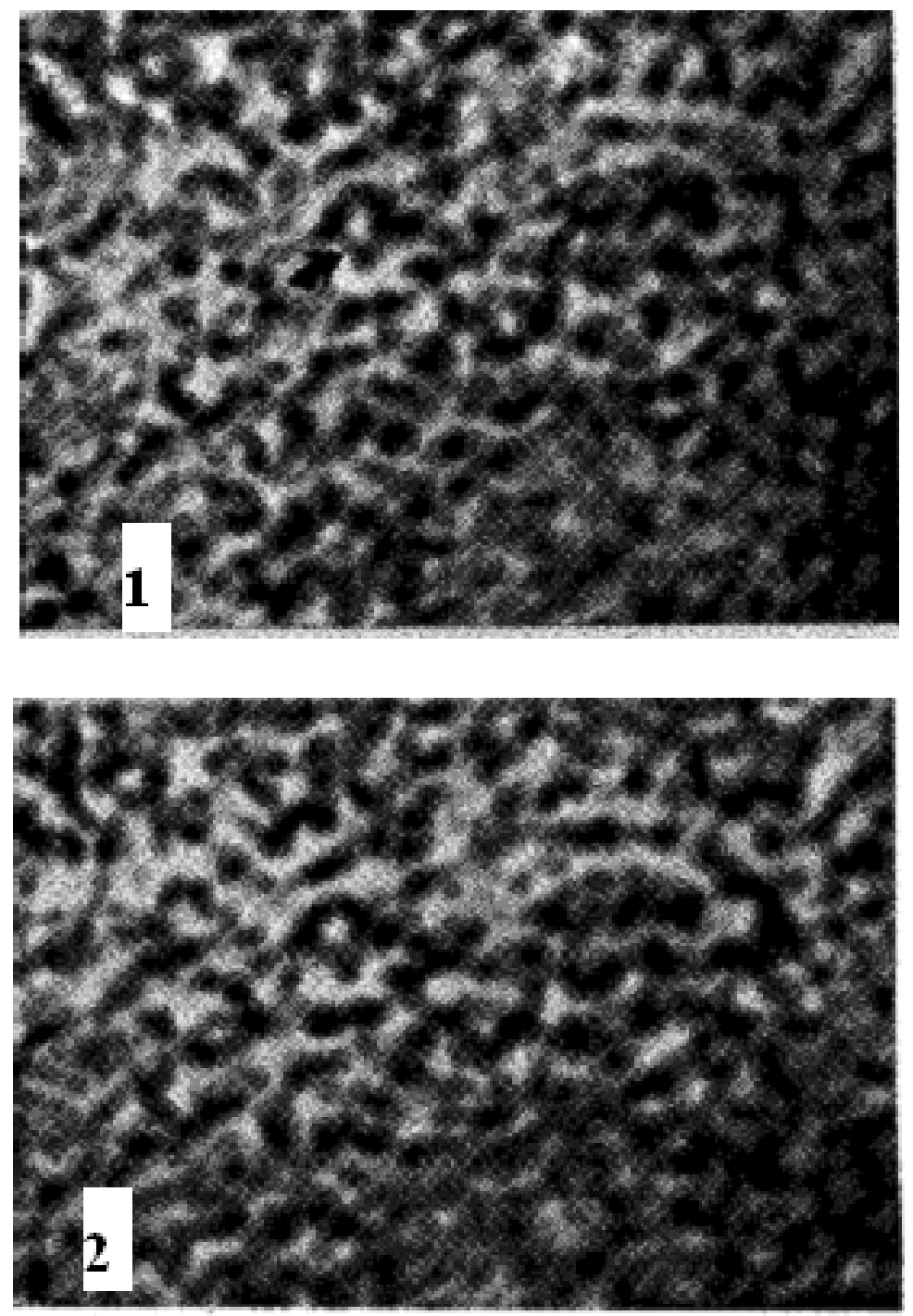

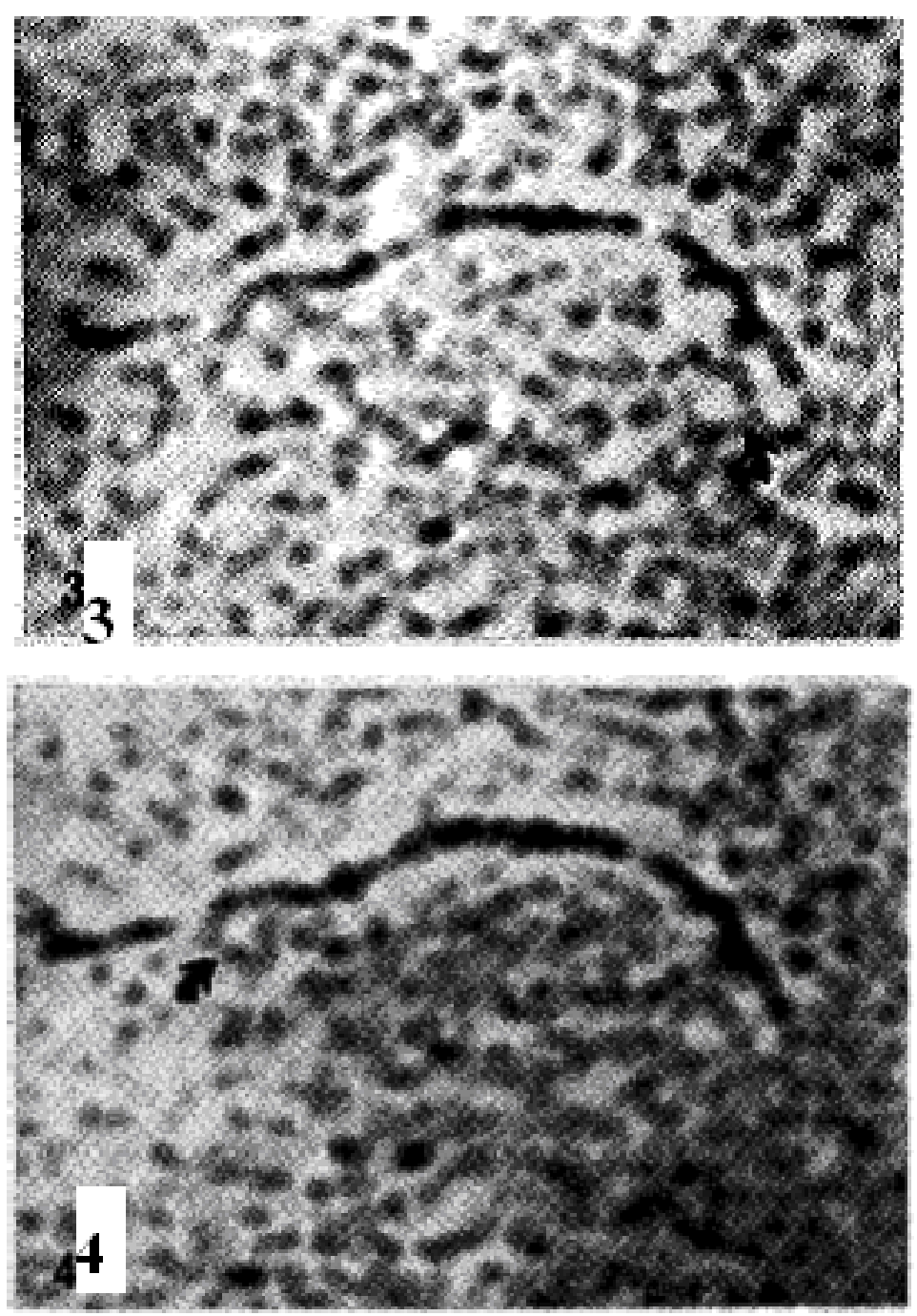

Figure 1. Plate (I) : Recorded Vertical Structures of the Filament. No. : $1234 \Delta \lambda$ Ao : 0.5 0.450 .40 .35 Scales : $1 \mathrm{~mm}=6.6 \mathrm{sec}$ of arc. 

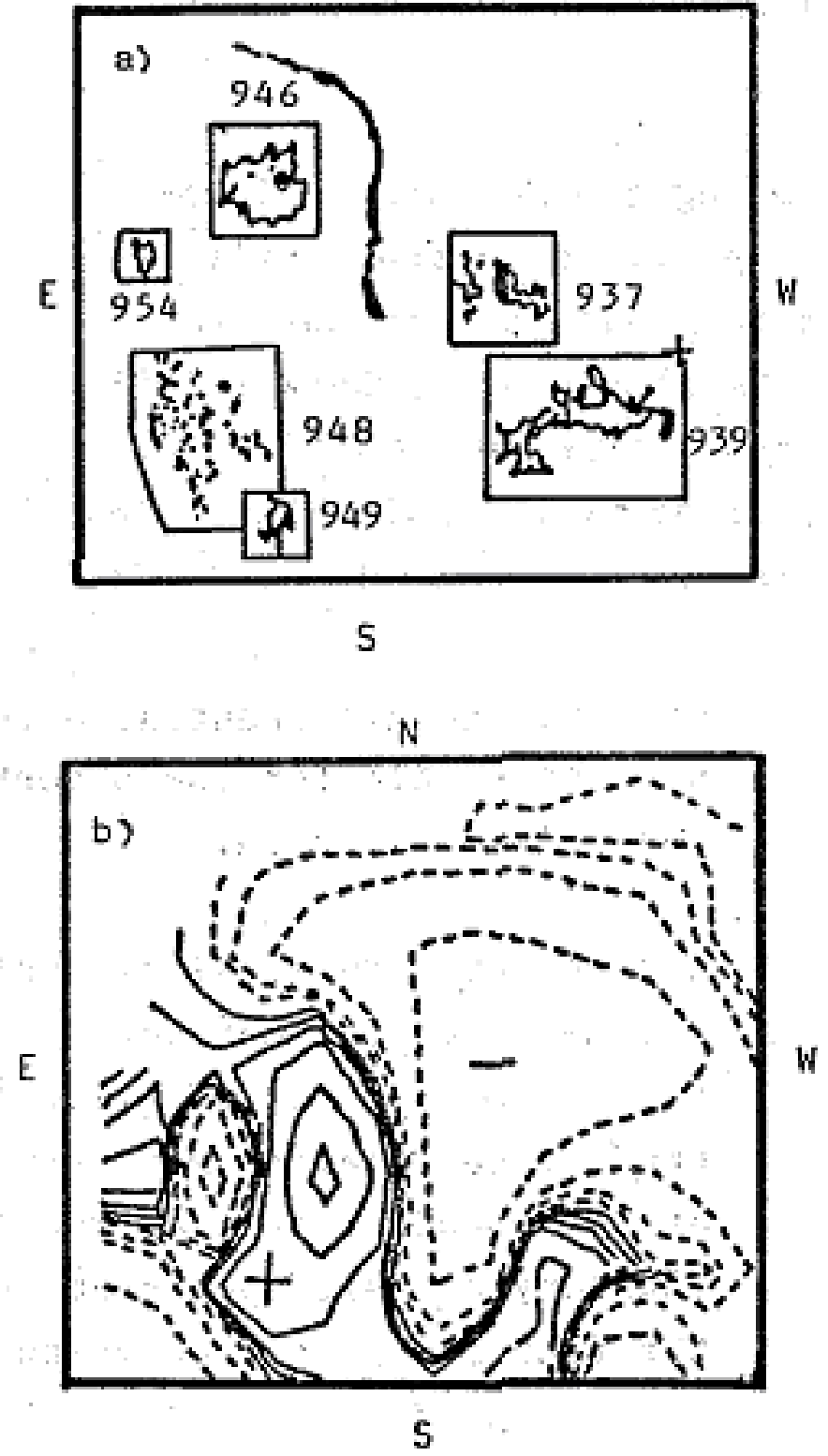

Figure 2. Fig 1-(a) Distribution of Active Regions around the Filament (23rd October, 14:31 UT) (b) Inversion of Polarity in both sides of the Filament (22:06 UT). 


\section{References}

Athay R. G. 1976, The Solar Chromesphere and Corona: Quiet Sun, Reideal, and Dordrecht

Bonnet R. M., Delache Ph. 1976, The Energy Balance and Hydrodynamics of The Solar Chromosphere and Corona Proc. IAU Coll. no. 36, G. de Bussac, Chermont- Ferrand

Bray R. J. and Laughead R. E. 1974, The Solar Chromosphere Chapman \& Hall, London

Domingo V., Fleck B. and Poland A. I. 1995, Solar Phys. 162, 1

Fleck B., Domingo V., and Poland A. I. 1995, The SOHO Mission, Solar Phys. 162, no 1-2

Fleck B. 1999, Proc. Summerschool and Workshop, Kanzelhohe Kluber Academic Publisher, Dordrecht, Netherlands, p. 1

Forbes T. 1986, Coronal and prominence plasmas NASA Conference publ. 2442, 21

Furth H. P., Killen J., Rosenbluth N.M. 1963, Phys. Fluids 6, 459

Galal A. A. 1983, HIAG Bull., III Seria A, 43

Galal A. A. 1989, Hvar OBS. Bull. 13, $401-411$

Giovanelli R.G. 1984, Secrets of the Sun 66, Cambridge University Press

Harison R. A., Sawyer E. C., Carter M. K. et al. 1995, Solar Phys. 162,233

Herman L. M. and Martin S. F. 1986, Coronal and Prominence Plasmas, NASA Conference publ. 2442,369

Howard R. 1959, ApJ 130, 193

Kenneth R. Lang 2001, The Cambridge Encyclopedia Of The Sun Cambridge University Pre

Martin S. F 1963, Solar Phys. 31, 3

Martin S. F 1986, Coronal and Prominence Plasmas, NASA Conferencepubl. 2442, 63

Plocieniak, S., and Rompolt, B. 1972, Solar Phys. 29, 399

Rompolt, B., And Bodgan, T. 1986, Coronal and Prominence plasmas, NASA Conference Publ. 2442,81

Smith, S.F. 1968, Structure and Development of Active Regions K.O. Kiepenheuer, 267

Sturrock, P.A. 1968, Structure and Development of Active Regions K.O. Kiepenheuer, 471

Ulmschneider P., Priest E. R. and Rosner R. 1991, Choromesphere and Coronal Heating Mechanisms Proc. Intern. Conference, Heidelberg 5-8 June (1990), Springr Heidelberg

VIRGO group 1995, VIRGO purple book v. 2.1

Zirin H. 1966, The Solar Atmosphere Blaisdell publishing Company, Waltham Mass, Toronto London, (Russian Copy, 348, Izdatelstvo Mir, Moscow, (1969)) 